\title{
Electrospun composite mats of alginate with embedded silver nanoparticles
}

\section{Synthesis and characterization}

\author{
Maila Castellano $^{1} \cdot$ Marina Alloisio $^{1} \cdot$ Rimah Darawish $^{1} \cdot$ Andrea Dodero $^{1} \cdot$ Silvia Vicini $^{1}$ \\ Received: 31 July 2018 / Accepted: 14 December 2018/Published online: 2 January 2019 \\ (C) Akadémiai Kiadó, Budapest, Hungary 2019
}

\begin{abstract}
In this work, a nanocomposite material based on alginate (Alg) and silver nanoparticles (AgNPs) was prepared by the synthesis "in situ" of the metal cores in the polymer solution through a wet, bottom-up approach. The formation of AgNPs within the alginate matrix (Alg-Ag) was confirmed by spectroscopic and morphological investigation. The nanocomposite material was then employed in combination with poly(ethylene oxide) for the production of crosslinked alginate membranes by electrospinning technique, following a simple and effective protocol optimized to obtain nano- to microscaled fibers. Field emission scanning electron microscopy (FESEM) studies on the composite membranes indicated that AgNPs of slightly increased dimensions are well distributed in the electrospun mats, thus proving that the electrospinning process does not significantly alter the morphology of the metal nanofillers and can be successfully adopted for the fabrication of nanofibers. In view of their potential applications in biomedical fields and food industry as antibacterial material, the nanocomposite mats were tested against thermal and UV sterilization, anti-mold treatment $(\mathrm{NaClO})$ and environmental changes $(\mathrm{pH})$ and their stability was monitored by means of thermogravimetric (TGA) and morphological (FESEM) techniques.
\end{abstract}

Keywords Sodium alginate $\cdot$ Silver nanoparticles $\cdot$ Nanocomposite mats $\cdot$ Electrospinning $\cdot$ Thermogravimetric analysis

\section{Introduction}

Polysaccharide-based nanocomposites, constituted by nanoscale fillers incorporated into a polysaccharide matrix, have achieved increasing importance in both academic and industrial world as green alternative to synthetic polymers. Because of their versatile properties, including biocompatibility, biodegradability, environmental safety and antimicrobial activity, they have been widely used in biomedicine as well as in a variety of industrial applications demanding biodegradable materials $[1,2]$. As an example, edible and biodegradable films

Electronic supplementary material The online version of this article (https://doi.org/10.1007/s10973-018-7979-z) contains supplementary material, which is available to authorized users.

Marina Alloisio

marina.alloisio@unige.it

1 Dipartimento di Chimica e Chimica Industriale, Università di Genova, Via Dodecaneso 31, 16146 Genoa, Italy of polysaccharide nanocomposites have been exploited in food packaging, due to their well-known gas barrier capabilities [3]. Moreover, being based on polymers deriving from renewable sources that are abundant in nature, they can be considered quite economic and easily recovered materials.

Among the most commonly employed polysaccharides in application purposes, sodium alginate (SAlg), a natural polymer extracted from brown algae, has acquired great relevance [4]. The reasons for this success are its biocompatibility, renewability, low cost and the ability to create gel in the presence of bivalent ions [5]. This property is directly correlated with its composition. As shown in Fig. 1, an alginate (Alg) chain is made of $(1,4)$-linked $\beta$-D-mannuronic acid $(\mathrm{M})$ and its $\mathrm{C}$-5 epimer $\alpha$-L-guluronic acid $(\mathrm{G})$, connected to each other in long sequences of M-Blocks, G-Blocks and MG-Blocks [6, 7]. The mechanism that regulates the gelling capacity of the polysaccharide is conditioned by the amount of G-Blocks present along the chain. The gelling proprieties are explained by the "eggbox" model, where the G-Blocks form cavities, which work as binding sites for bivalent cations and arrange 
Fig. 1 Structural formula of alginate

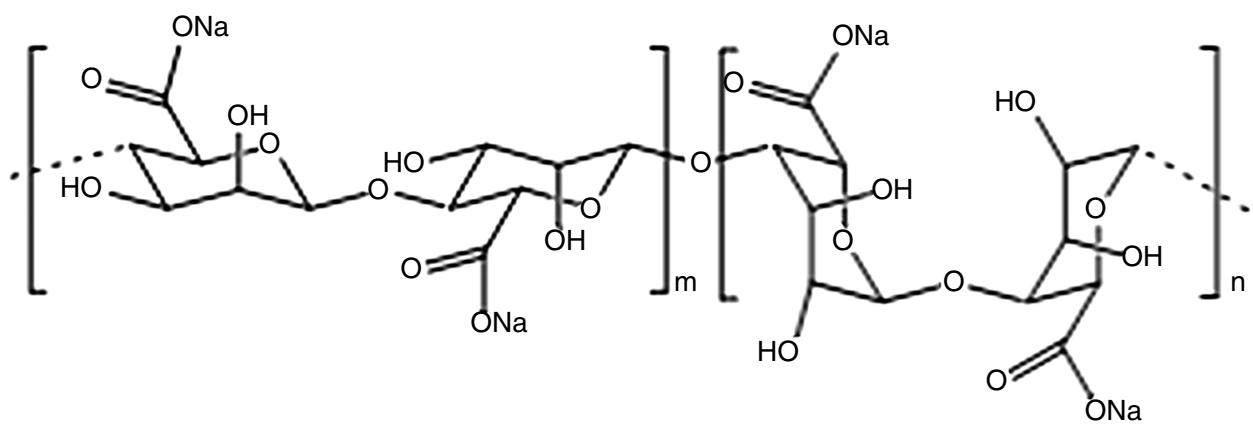

themselves all around [8]. This reaction is almost instantaneous, and the stability of the gel depends on the kind and quantity of the ions added to the alginate solution as well as on the length of the G-Blocks for the formation of stable junctions [9, 10]. Due to its gelling properties under mild conditions, SAlg has been widely applied in biomedical areas, especially in tissue engineering [11] despite the low mechanical properties of the polymer. In order to obtain mechanically robust hydrogels, SAlg matrices have been recently added with hydroxyapatite [12], cellulose nanocrystals, and halloysite nanotubes [13] and graphene oxide [14]. Moreover, it has been demonstrated that additional antimicrobial activity can be successfully conferred to the scaffold by adding metal nano-reinforcements to the polysaccharide bulk [15]. In particular, a non-cytotoxic nanocomposite, which has been proved to be very effective against Gram + and Gram- bacteria, has been produced by embedding silver nanoparticles (AgNPs) in chitosan and/or alginate matrices through facile procedures $[16,17]$.

The properties of the final material also depend on the fabrication processing. A large number of techniques are available, including in situ preparations, film casting, layerby-layer assembly and electrospinning. Electrospinning [18-23] is a simple, versatile and cost-effective technique to obtain flexible nano- to microscaled fibers from a wide variety of polymer solutions and polymer melts. By this technology, nonwoven membranes are produced from a fine jet of liquid subjected to a proper electric potential [24]. Depending on the specific polymer used, a range of desirable fabric properties, such as strength, thermal and mechanical properties, can be achieved in a one-step process. However, electrospinning from aqueous solutions of biopolymers, and polysaccharides in particular, is difficult because of the high viscosity, the ionic character and, above all, the tendency to form gels via H-bonding among the polymeric chains. So far, the technique was successfully applied for collagen, alginate, hyaluronic acid and chitosan by blending with non-toxic and biocompatible synthetic polymers such as poly(ethylene oxide) (PEO), poly(vinyl pyrrolidone) (PVP) or poly(vinyl alcohol) (PVA) [25-28], which can improve the processability of the biopolymers without affecting their biocompatibility.
Starting on these bases, we aimed at preparing a polysaccharide-based nanocomposite able to be electrospun in form of nanofibers for biomedical applications and food industry. The choice fell on SAlg incorporated with AgNPs generated "in situ" through a wet, bottom-up approach previously set up by us [31]. Once obtained, the nanocomposite was blended with PEO and processed by electrospinning technique by taking advantage of our expertise in manufacturing polysaccharide-based materials. Although AgNPs-incorporating alginate was already exploited in form of porous sponges [29] or spinned fibers [30], to name a few, this work represents the first attempt to produce nonwoven membranes of this nanocomposite by means of electrospinning technique, as far as we know.

The effect of the metal nanofillers on the fabrication process was studied through morphological (FESEM) and spectroscopic techniques. Moreover, in view of their potential employment as biodegradable scaffolds or packaging membranes, the mats stability toward sterilization procedures, anti-mold treatments and environmental changes was also evaluated. In particular, the nanofibers behavior under physical (heating, UV exposure) and chemical (acidic, alkaline and oxidative media) perturbations was investigated by means of thermal (thermogravimetric analysis, TGA) and morphological (FESEM) characterization.

\section{Experimental}

\section{Materials}

SAlg, with $\overline{\mathrm{M}}_{\mathrm{v}}$ around $200,000 \mathrm{~g} \mathrm{~mol}^{-1}$ and composed of approximately $39 \%$ mannuronic and $61 \%$ guluronic acid $(\mathrm{G} / \mathrm{M}$ ratio $=1.56)$, purchased from FMC Biopolymers (Norway), was used.

PEO with $\overline{\mathrm{M}}_{\mathrm{v}}$ of $600,000 \mathrm{~g} \mathrm{~mol}^{-1}$ and sodium borohydride $\left(\mathrm{NaBH}_{4}\right)$ purchased from Sigma-Aldrich were used.

Silver nitrate $\left(\mathrm{AgNO}_{3}\right)$, purchased from Merck, was used. Triton X-100, nonionic surfactant of 4-(1,1,3,3-tetramethylbutyl)phenyl-polyethylene glycol, t-octylphenoxy 
polyethoxyethanol and polyethylene glycol tert-octylphenyl ether at laboratory grade, purchased by Sigma-Aldrich, were used.

Barium chloride dihydrate at reagent grade (assay $\geq 99 \%$ ) purchased from Sigma-Aldrich was used. Absolute ethanol, denatured with $1 \% \mathrm{v} / \mathrm{v}$ of cyclohexane (assay $\geq 99.8 \%$ ), was purchased from Sigma-Aldrich.

All chemicals were used without any further purification. The aqueous solutions were made using deionized water.

\section{Synthesis of silver nanoparticles-alginate nanocomposite (Alg-Ag)}

Silver nanoparticles (AgNPs) stabilized within sodium alginate matrix were obtained at room temperature through a chemical reduction of silver nitrate in an aqueous solution of the polysaccharide by following a synthetic protocol previously set up with chitosan [31]. In a typical preparation, $100 \mathrm{~mL}$ of 4 mass\% aqueous solution of SAlg was added of a proper aliquot $(1 \mathrm{~mL})$ of the salt precursor $\left(\mathrm{AgNO}_{3}, 60 \mathrm{mmol} \mathrm{L}^{-1}\right)$ and mixed for $1 \mathrm{~h}$; then, a 3-h aged aqueous solution of $\mathrm{NaBH}_{4}\left(1 \mathrm{~mL}, 60 \mathrm{mmol} \mathrm{L}^{-1}\right)$ was introduced drop by drop, maintaining the stirring for approximately $2 \mathrm{~h}$ to ensure full reaction. The color change in the mixture from light brown to dark brown with greenish shades indicated the formation of AgNPs within the alginate matrix, as confirmed by UV-Vis characterization. Once prepared, the colloidal solution was kept at $4{ }^{\circ} \mathrm{C}$ to avoid biodegradation without further purification.

\section{Membranes preparation by electrospinning}

PEO and SAlg were separately dissolved in deionized water for $2 \mathrm{~h}$ at room temperature under continuous stirring to obtain 4 mass\% solutions. Mixtures of PEO with SAlg and PEO with $\mathrm{Alg}-\mathrm{Ag}$ of ratio 50:50 were stirred till a homogeneous system was formed. Then, a 1 mass\% Triton $\mathrm{X}-100$ was added to the system to improve the formation of nanofibrous structures. After removing air bubbles by resting, the mixtures were stored in fridge overnight.

The electrospinning apparatus is constituted by a $5-\mathrm{mL}$ syringe containing the polymeric solution, a volumetric pump that allows the release of the solution from the syringe at a constant flow rate, an electrically grounded collector for the fibers and a high voltage generator that produces the electric field required for the process.

Solutions were pumped inside a Teflon pipe, which had a filter and a needle (connected to the cathode) on the extremity, placed over a round copper collector $(13 \mathrm{~cm} \varnothing)$ connected to the anode. It was possible to change three main operative parameters: the distance between the needle and the anode, the flow rate applied to the solution by the volumetric pump and the voltage (ddp). In our experiments, performed at $25^{\circ} \mathrm{C}$, the distance between the needle and the collector (anode) was $15 \mathrm{~cm}$ and the ddp set at 11 and $15 \mathrm{kV}$ for the $\mathrm{PEO} / \mathrm{Alg}$ and the $\mathrm{PEO} / \mathrm{Alg}-\mathrm{Ag}$ systems, respectively, maintaining the flow rate at $0.5 \mathrm{~mL} \mathrm{~h}^{-1}$. In order to monitor the electrospinning process, micrographs of the Taylor's cone were acquired with a computer-controlled digital camera (Moticam10). In wet conditions, the copper collector was placed inside a plastic cup containing the coagulation solution, made of a $60: 40$ water/EtOH mixture with 3 mass $\%$ of $\mathrm{BaCl}_{2}$. In order to eliminate the excess of barium salt, the membranes were then washed for $1 \mathrm{~h}$ in 60:40 water/EtOH mixture. Hence, the membranes were dried in air and kept at room temperature.

\section{Study of stability}

Squared membranes of $\mathrm{PEO} / \mathrm{Alg}$ and $\mathrm{PEO} / \mathrm{Alg}-\mathrm{Ag}$ with an area of $2 \times 2 \mathrm{~cm}^{2}$ were tested to assess the resistance to sterilization and chemical treatments in the following conditions:

(a) resistance to thermal sterilization: membranes were heated in oven at $120^{\circ} \mathrm{C}$ for $1 \mathrm{~h}$;

(b) resistance to UV light sterilization: membranes were subjected to UV light for $10 \mathrm{~min}$ and $1 \mathrm{~h}$ by using a Rayonet photochemical chamber reactor, operating at $254 \mathrm{~nm}$ and $35 \mathrm{~W}$ power, by keeping the membranes at a $10-\mathrm{cm}$ distance from the UV lamps;

(c) resistance to oxidation: membranes were soaked for $16 \mathrm{~h}$ in $\mathrm{NaClO}$ aqueous solution ( 5 mass \%);

(d) resistance to acid and alkaline treatments: membranes were soaked for $16 \mathrm{~h}$ in $1 \mathrm{molL}^{-1} \mathrm{HCl}$ solution $\left(\mathrm{pH}\right.$ 1) and $1 \mathrm{molL}^{-1} \mathrm{NaOH}$ solution $(\mathrm{pH}$ 13), respectively.

\section{Characterization methods}

\section{UV-Vis spectroscopy}

Electronic absorption spectra at room temperature were recorded on a PerkinElmer Lambda 9 spectrophotometer, equipped with an integrating sphere for the characterization of the mats.

\section{Fourier transform infrared spectroscopy}

FTIR spectra were collected on untreated membranes by means of a Bruker Vertex 70 instrument, operating in ATR mode. 


\section{Field emission scanning electron microscopy}

A morphological investigation of both colloids and mats was carried out using a Field Emission Scanning Electron Microscope ZEISS SUPRA $40 \mathrm{VP}$, operating at $20 \mathrm{keV}$ in both direct (in-lens mode) and back-scattered (QBSD mode) configuration. All the samples were thinly sputtercoated with carbon using a Polaron E5100 sputter coater, to obtain good conductivity. In order to evaluate the size of both nanoparticles and fibers, an image analysis was performed. No less than 200 measurements were elaborated from SEM images at different magnifications with the open-source software ImageJ. Recorded values were processed with two different statistical tests: the KolmogorovSmirnov test [32] to verify the population and the theoretical continuous distribution, applicable to a Gaussian distribution, and the median absolute deviation (MAD) test [33] to verify the presence of outliers inside the collected data. After these preliminary tests, the values were processed to evaluate the Gaussian distribution of the experimental data using the ordinary least squares (OLS) in regression and obtain an optimized distribution curve.

\section{Thermogravimetric analysis (TGA)}

TGA profiles were acquired by means of a Mettler Toledo TGA/DSC1 STARe System. The measurements were taken on about $5 \mathrm{mg}$ of each sample, in dynamic mode at a heating rate of $10{ }^{\circ} \mathrm{C} \mathrm{min}^{-1}$, operating in the range $120-700{ }^{\circ} \mathrm{C}$ under nitrogen atmosphere (gas flow of $80 \mathrm{~mL} \mathrm{~min}^{-1}$ ) and in the range $700{ }^{\circ} \mathrm{C}$ to $900{ }^{\circ} \mathrm{C}$ under air (gas flow of $80 \mathrm{~mL} \mathrm{~min}^{-1}$ ). The samples were dried by heating at $120{ }^{\circ} \mathrm{C}$ for $1 \mathrm{~h}$ before use in order to remove the residual moisture and physically absorbed water.

\section{Results and discussion}

\section{Characterization of Alg-Ag nanocomposite}

FESEM images of an aqueous suspension of AgNPs protected by alginate chains are displayed in Fig. 2, showing the presence of well-separated silver cores randomly assembled in aggregates of $200-500 \mathrm{~nm}$ dimensions. The AgNPs exhibit nearly spherical or quite elongated (aspect ratio $=1.3 \pm 0.3$ ) shape. The histogram of the size distribution, reported below the images, indicates that the particles have an average diameter of about $22.0 \mathrm{~nm}$ and limited size dispersion $(5.7 \mathrm{~nm})$.

In agreement with this result, the spectral profile of the colloidal suspension, reported in Fig. 3, is characterized by a well-defined peak at $400 \mathrm{~nm}$, corresponding to the
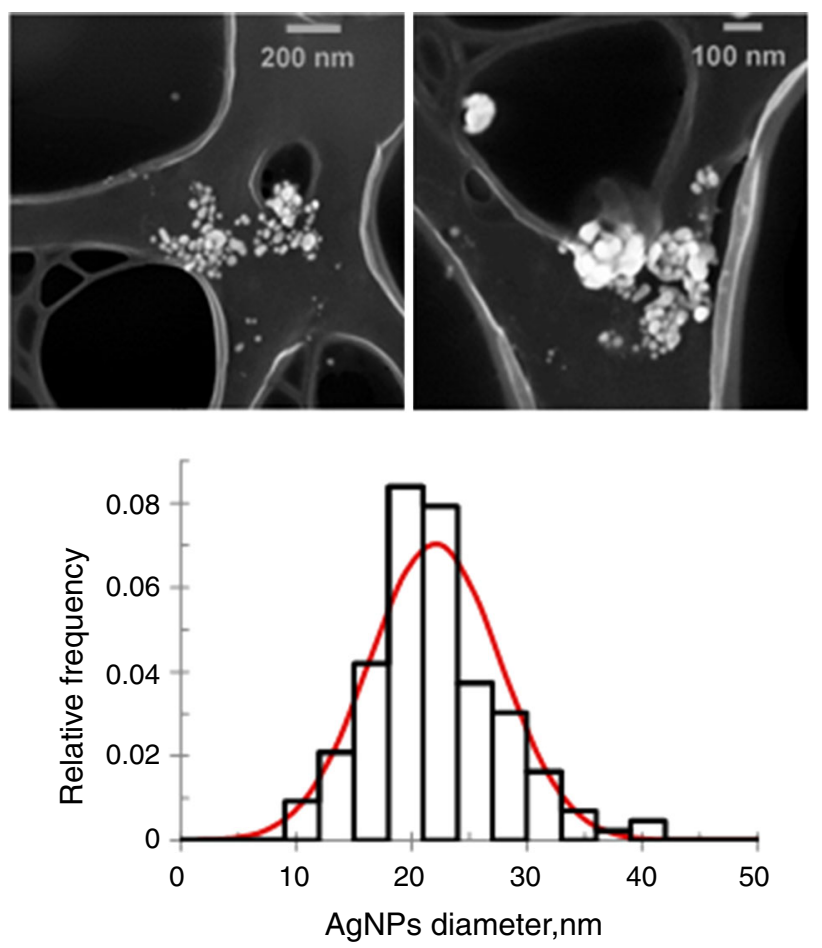

Fig. 2 FESEM images collected in direct configuration (in-lens mode) of AgNPs in Alg-Ag nanocomposite (top); the corresponding core size distribution histogram is reported

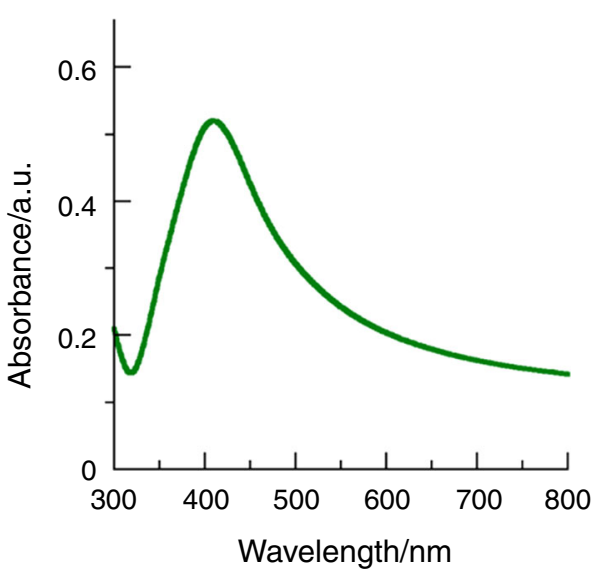

Fig. $3 \mathrm{UV}-\mathrm{Vis}$ spectrum of a fresh aqueous suspension of $\mathrm{Alg}-\mathrm{Ag}$

surface plasmon resonance (SPR) band typical of quite homogeneous silver nanoparticles.

UV-Vis technique was also employed to monitor AlgAg stability with time. Absorption spectra of the sample recorded over a period of 2 months (Fig. 1S in electronic supplementary material) highlighted little changes in both lineshape and position of the SPR band, indicating that the silver colloids are quite stable in the polysaccharide solution. This result confirms that alginate proved to be an effective protecting agent in the wet synthesis of silver nanostructures by means of chemical approaches. 


\section{Characterization of the electrospun mats}

$\mathrm{PEO} / \mathrm{Alg}$ and $\mathrm{PEO} / \mathrm{Alg}-\mathrm{Ag}$ solutions showed good spinnability properties. The wet conditions, consisting of a solution containing divalent ions, in which the newly formed fibers are collected, allow to directly cross-link the alginate during the membrane formation. Therefore, the coagulation solution ( 3 mass $\%$ of $\mathrm{BaCl}_{2}$ in $60: 40$ water/ EtOH) was created for two purposes: (1) to cross-link alginate by means of the barium salt, which worked as a gelling agent and was selected on the basis of its affinity with alginate, i.e., $\mathrm{Ba}^{2+}>\mathrm{Ca}^{2+} \gg \mathrm{Mg}^{2+}$ [10]; (2) to avoid fibers dissolving in the aqueous environment. The water/ethanol mixture was selected because ethanol is a non-solvent for the alginate [34] and the 60:40 ratio represents an appropriate compromise as it allows dissolving enough barium salt and not dissolving the alginate.

After rinsing and drying, the membranes had a tissuelike appearance and were easy to handle. Figure 4 displays the FESEM images of the composite PEO/Alg-Ag mats (top) and their fully polymeric counterparts $\mathrm{PEO} / \mathrm{Alg}$ mats (bottom). The images showed the formation of well-defined nanofibers. The fiber diameters were in nanometer scale with a mean value of around $100-150 \mathrm{~nm}$, regardless of the presence of AgNPs in the initial solution; therefore, embedding silver nanoparticles in the alginate matrix did not modify the electrospinning process of the polymeric solution.
As far as the morphology of the silver colloids within the membranes is concerned, FESEM image acquired at higher magnification shows almost spherical-like nanostructures of average diameter of about $40 \mathrm{~nm}$, corresponding to a nearly $50 \%$ size increase of the pristine AgNPs (Fig. $2 \mathrm{~S}$ in electronic supplementary material). It is likely that the electrospinning process induced a partial coagulation of the silver nuclei, which, however, did not lose their nanoscale dimensions. The nanofillers were found to be embedded in the polysaccharide matrix or disposed in aggregates outside the fibers. The Ag signal in the EDS (energy-dispersive spectroscopy) analysis (Fig. 3S in electronic supplementary material) supports the hypothesis that the observed nanostructures correspond to AgNPs.

Once again, UV-Vis characterization of the mats (Fig. 5) proved to be in agreement with the morphological data. The spectrum of the membrane containing embedded silver nanoparticles exhibits two broad absorption bands centered around 450 and $650 \mathrm{~nm}$. Although poorly resolved, the higher energy band is resembling that found for isolated AgNPs in Fig. 3, whereas the band at longer wavelengths can be ascribable to aggregates of silver nanoparticles, thus confirming the presence of both dispersed and aggregated $\mathrm{Ag}$ cores in the composite membrane. As expected, the spectral lineshape of the PEO/Alg membrane is featureless, owing to the absence of specific
Fig. 4 FESEM images collected in direct configuration (in-lens mode) of $\mathrm{PEO} / \mathrm{Alg}-\mathrm{Ag}$ (top) and $\mathrm{PEO} / \mathrm{Alg}$ (bottom) electrospun mats
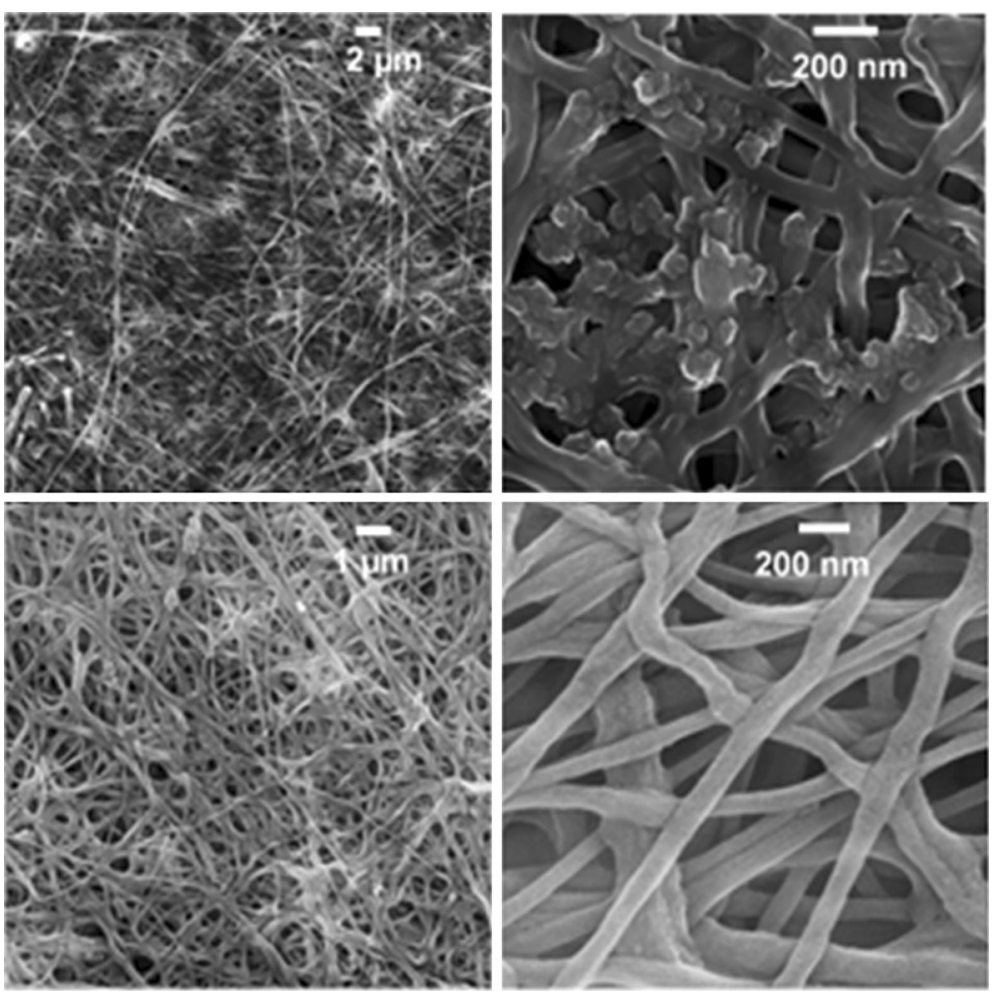


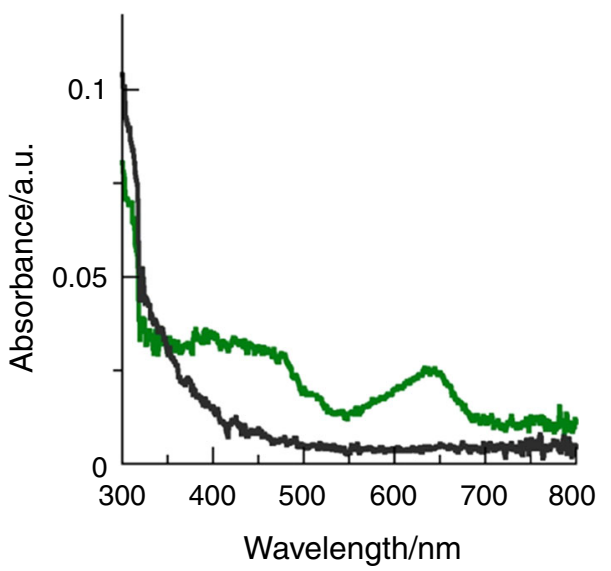

Fig. $5 \mathrm{UV}-\mathrm{Vis}$ spectra of $\mathrm{PEO} / \mathrm{Alg}-\mathrm{Ag}$ (green line) and $\mathrm{PEO} / \mathrm{Alg}$ (black line) untreated mats fabricated by electrospinning technique under the same experimental conditions

absorptions of the two polymers in the visible spectral range.

$\mathrm{PEO} / \mathrm{Alg}-\mathrm{Ag}$ mats were also investigated by means of FTIR technique. The infrared spectrum collected in ATR mode is reported in Fig. 6 together with those of SAlg and PEO in powders to facilitate the data interpretation. The assignment of main bands to characteristic vibrations according to the literature [35-39] is given in Table 1. The spectral profile of the composite membrane mostly resembles that of the polysaccharide, as the dotted lines drawn in correspondence of the main signatures clearly highlight. However, some variations can be observed, briefly listed below, which suggest the presence of AgNPs:

- The intense, enlarged band around $3400 \mathrm{~cm}^{-1}$ associated with $\mathrm{O}-\mathrm{H}$ stretching vibration looks sharpen and more structured in the case of PEO/Alg-Ag mats. Since this type of band lineshape was already found in alginate-based composites reinforced with nanofillers [40], this features change can be associated with the presence of AgNPs linked to the polysaccharide chains;

- The asymmetric carboxyl stretching $\left(\mathrm{v}_{\mathrm{a}}\left(\mathrm{COO}^{-}\right)\right.$is split into two for the composite membrane, in that the peak at $1595 \mathrm{~cm}^{-1}$, typical of the free polysaccharide, shows a shoulder at higher wavenumber $\left(1630 \mathrm{~cm}^{-1}\right)$. As upshifts of $\mathrm{v}_{\mathrm{a}}\left(\mathrm{COO}^{-}\right)$mode are usually attributed to the establishment of intermolecular interactions involving the carboxylic groups [37], this result can denote that AgNPs are actually anchored to Alg chains by means of the polysaccharide $\mathrm{COO}^{-}$substituents.

Conversely, no significant evidences of the PEO component were detected in the nanocomposite mats, at least as far as the membrane surface is concerned. Indeed, also the vibrational modes common to both polymers, such as the ether bond stretching $((v(\mathrm{C}-\mathrm{O}-\mathrm{C}))$ around

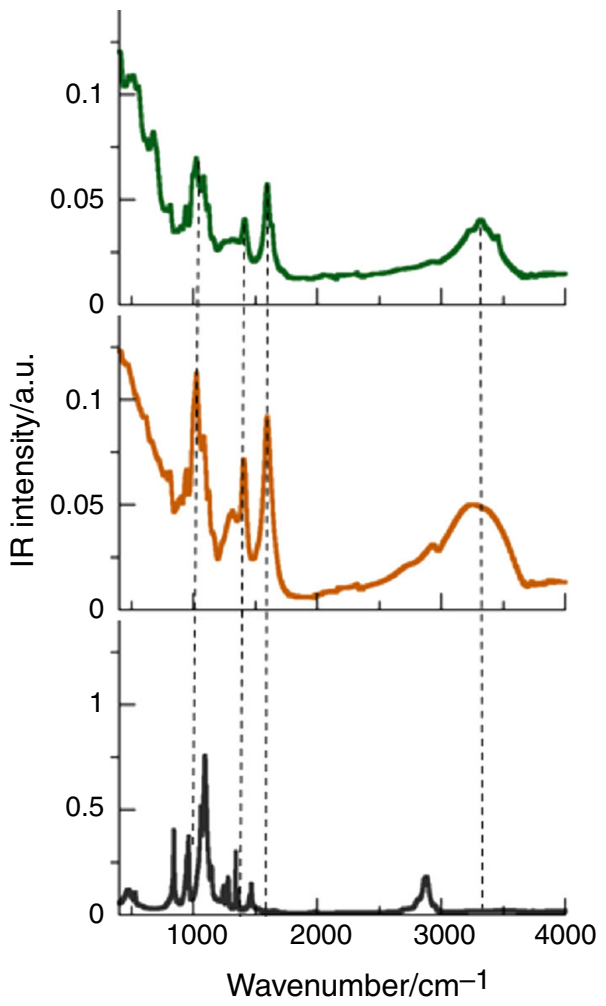

Fig. 6 From top to bottom: FTIR-ATR spectra of PEO/Alg-Ag mats, SAlg powders and $\mathrm{PEO}$ powders

$1080-1090 \mathrm{~cm}^{-1}$, show wavenumbers typical of polysaccharides.

Overall, although the presence of AgNPs cannot be directly detected by FTIR investigation [41], the technique provided an indirect confirmation of the metal nanoparticles encapsulation within the Alg matrix.

Both composite and all-polymeric mats were also characterized by thermogravimetric analysis in order to achieve quantitative information on their composition and on the influence exerted by AgNPs on the nanofiber stability. With the aim of removing the residual moisture and physically absorbed water, the samples were previously dried by heating at $120{ }^{\circ} \mathrm{C}$ for $1 \mathrm{~h}$. Figure 7 shows the mass loss (TG) curves obtained from $\mathrm{PEO} / \mathrm{Alg}-\mathrm{Ag}$ (solid green line) and $\mathrm{PEO} / \mathrm{Alg}$ (solid black line) subjected to the combined nitrogen/air thermal cycle detailed in "Experimental" section. Derivative (DTG) profiles are also reported to better highlight the degradation steps. Both TG curves indicate two main stages of decomposition in nonoxidative atmosphere, approximately located at the degradation temperatures $\left(T_{\mathrm{d}}\right)$ of $280{ }^{\circ} \mathrm{C}$ and $415{ }^{\circ} \mathrm{C}$. According to the literature [30,42-45], these values correspond to the thermal disruption of the glycosidic bonds of alginate and to the decomposition of PEO, respectively. The smoother dropping stages observed below $200{ }^{\circ} \mathrm{C}$ and $700{ }^{\circ} \mathrm{C}$ are also attributable to the alginate degradation, which is 
Table 1 Spectroscopic data extracted from FTIR-ATR spectra of Fig. 6

\begin{tabular}{llll}
\hline Vibrational mode & PEO/Alg-Ag mats & SAlg powders & PEO powders \\
\hline$v(\mathrm{O}-\mathrm{H})$ & 3454 & $\sim 3250$ & - \\
& 3318 & & - \\
& 3246 & & \\
$v_{\mathrm{a}}\left(\mathrm{COO}^{-}\right)$ & 1630 & 1594 & 1467 \\
& 1595 & & - \\
$\delta(\mathrm{C}-\mathrm{H})$ & - & - & 1341 \\
$v_{\mathrm{s}}\left(\mathrm{COO}^{-}\right)$ & 1414 & 1406 & 1279 \\
$\delta\left(\mathrm{CH}_{2}\right)$ & - & - & 1241 \\
$\tau_{\mathrm{a}}\left(\mathrm{CH}_{2}\right)$ & - & - & 1093 \\
$\tau_{\mathrm{s}}(\mathrm{CH})$ & - & - & \\
$v(\mathrm{C}-\mathrm{O}-\mathrm{C})$ & 1083 & 1083 & \\
& 1054 & 1024 & 961 \\
& 1023 & & - \\
$v(\mathrm{C}-\mathrm{O})+\rho\left(\mathrm{CH}_{2}\right)$ & - & - & 841 \\
$v(\mathrm{C}-\mathrm{O})$ & 939 & 945 & \\
$(\beta-\mathrm{glycosidic} \mathrm{link})$ & 902 & 902 & - \\
$v(\mathrm{C}-\mathrm{O})$ & - & & \\
\hline
\end{tabular}

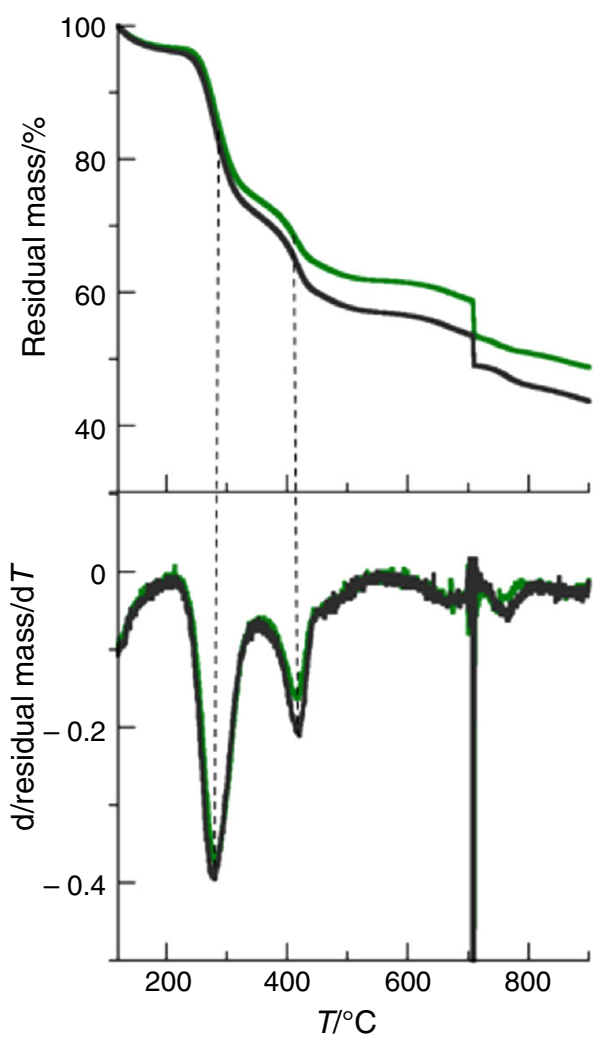

Fig. 7 TG (top) and corresponding DTG (bottom) curves of mats of untreated $\mathrm{PEO} / \mathrm{Alg}-\mathrm{Ag}$ (green lines) and $\mathrm{PEO} / \mathrm{Alg}$ (black lines). (Color figure online)

known to proceed through a multi-step mechanism [30]. In detail, the initial mass loss of about 3-4\% was usually associated with the release of water chemically linked to carboxylate groups through polar interactions [45] that cannot be eliminated by heating at $120{ }^{\circ} \mathrm{C}$. The mass loss in the $500-700{ }^{\circ} \mathrm{C}$ temperature range was instead ascribed to the further decomposition of the intermediate carbonaceous material deriving from partially degraded alginate [30]. It can be noticed that below $500{ }^{\circ} \mathrm{C}$ the TG profiles of the two samples are almost overlapped, suggesting that the presence of AgNPs did not affect the thermal properties of the two polymers, in particular as far as the stability of alginate is concerned. The switch from $\mathrm{N}_{2}$ atmosphere to air, marked at $700{ }^{\circ} \mathrm{C}$, caused the complete oxidation of the polysaccharide component, leaving a residual mass corresponding to the metal content (silver and barium for the $\mathrm{PEO} / \mathrm{Alg}-\mathrm{Ag}$ mats and only barium for the $\mathrm{PEO} / \mathrm{Alg}$ ones).

According to the TG and DTG curves interpretation, quantitative data are collected in Table 2 with the aim of providing the percentage composition of the nanofibers. The most remarkable variation is found for the residual mass that turned out to be increased from 43.7 to $48.8 \%$ in the composite mats. Assuming the barium quantity constant in both samples, the 5\% increment can be attributed to the silver content, thus confirming the presence of AgNPs within the polysaccharide matrix. The values corresponding to other components show less significant changes. As far as the polymeric moieties are concerned, it can be noticed that PEO percentage is considerably reduced with respect to the starting solutions, most likely owing to the partial dissolution of the synthetic polymer in the coagulation bath. This result is in agreement with the absence of PEO traces on the composite mats surface, already evidenced in the FTIR-ATR spectrum of Fig. 6. 
Table 2 Percentage composition of mats of PEO/SAlg-Ag and PEO/ SAlg from TGA profiles of Fig. 7

\begin{tabular}{llcl}
\hline Component & $\Delta T /{ }^{\circ} \mathrm{C}$ & $\mathrm{PEO} / \mathrm{Alg}-\mathrm{Ag} / \%$ & $\mathrm{PEO} / \mathrm{Alg} / \%$ \\
\hline $\mathrm{H}_{2} \mathrm{O}$ & $120-200$ & 3.2 & 3.8 \\
$\mathrm{PEO}$ & $200-350$ & 22.6 & 24.4 \\
$\mathrm{SAlg}$ & $350-900$ & 25.4 & 28.1 \\
total residue & 900 & 48.8 & 43.7 \\
$\mathrm{Ag} *$ & - & 5.1 & - \\
\hline
\end{tabular}

*Evaluated by subtracting the values of total residual of PEO/SAlg mats from that of $\mathrm{PEO} / \mathrm{SAlg}-\mathrm{Ag}$ mats

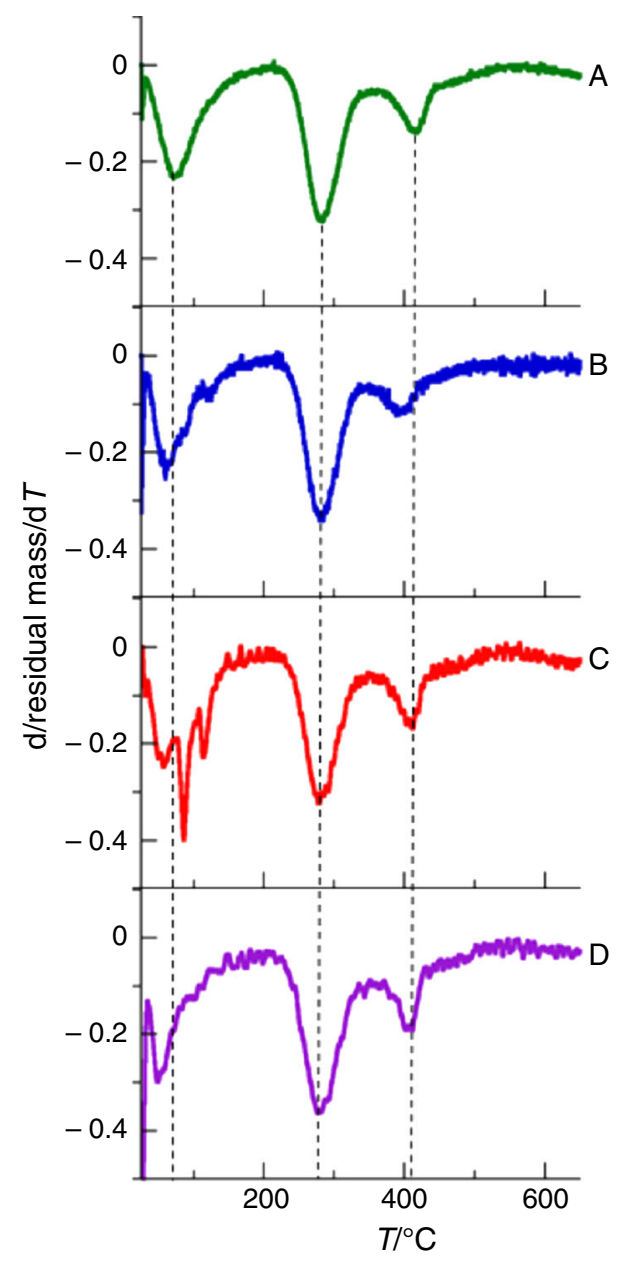

Fig. 8 DTG curves of mats of PEO/Alg-Ag before (green line A) and after physical treatments; heating at $120^{\circ} \mathrm{C}$ for $1 \mathrm{~h}$ (blue line B), UV irradiation for $10 \mathrm{~min}$ (red line $\mathrm{C}$ ) and for $1 \mathrm{~h}$ (purple line $\mathrm{D}$ ). (Color figure online)

\section{Stability tests of composite mats}

By taking into account the well-recognized antibacterial properties of silver nanoparticles, the most promising use of AgNPs-embedding alginate is for biomedical and food

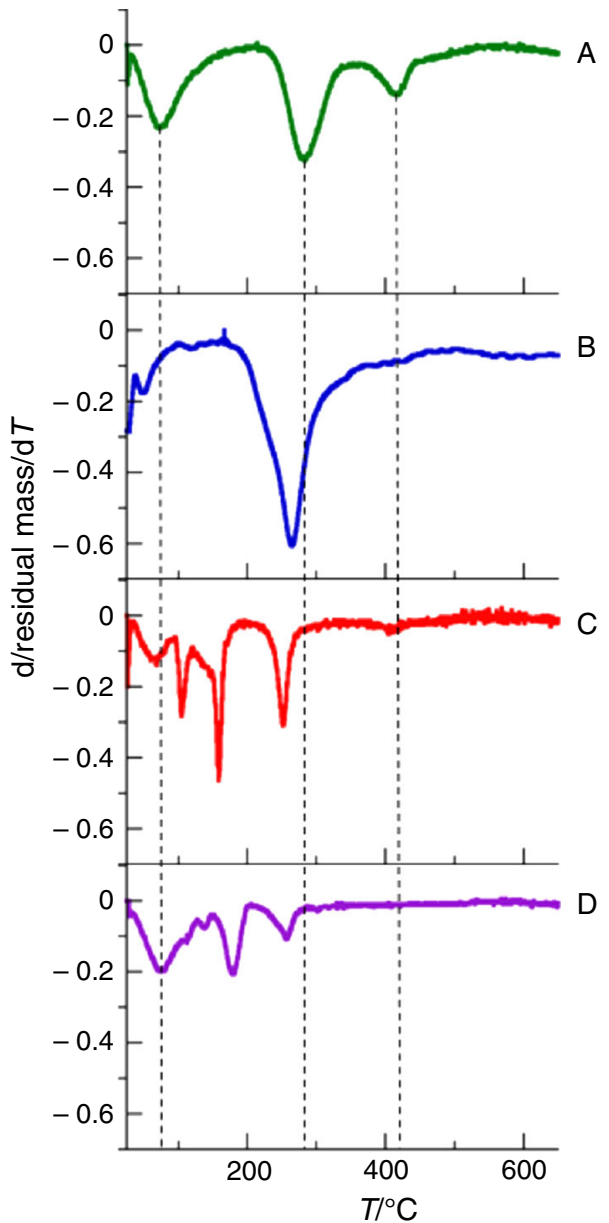

Fig. 9 DTG curves of mats of PEO/Alg-Ag before (green line A) and after chemical treatments; $\mathrm{HCl}, 1 \mathrm{~mol} / \mathrm{L}$ (blue line $\mathrm{B}$ ), $\mathrm{NaOH}, 1 \mathrm{~mol} /$ $\mathrm{L}$ (red line $\mathrm{C}$ ) and $\mathrm{NaClO}, 5$ mass\% (purple line D). (Color figure online)

Table 3 Stability against sterilization and environmental parameters of PEO/Alg/Alg@AgNPs and PEO/Alg

\begin{tabular}{llll}
\hline Treatments & $\begin{array}{l}T_{\mathrm{d}}{ }^{\circ} \mathrm{C} \\
\text { PEO/Alg }\end{array}$ & $\begin{array}{l}T_{\mathrm{d}} /{ }^{\circ} \mathrm{C} \\
\text { PEO/Alg-Ag }\end{array}$ & $\begin{array}{l}T_{5 \%} /{ }^{\circ} \mathrm{C} \\
\text { PEO/Alg-Ag }\end{array}$ \\
\hline Untreated sample & 277.6 & 280.9 & 269.5 \\
$T / 120{ }^{\circ} \mathrm{C}$ & 280.7 & 280.8 & 267.2 \\
$\mathrm{UV} / 10 \mathrm{~min}$ & 288.2 & 280.0 & 269.1 \\
$\mathrm{UV} / 1 \mathrm{~h}$ & 289.0 & 279.7 & 260.5 \\
$\mathrm{pH} \mathrm{1}$ & 263.3 & 264.3 & $217.0^{*}$ \\
$\mathrm{pH} \mathrm{13}$ & 249.6 & 250.5 & $152.8^{* *}$ \\
oxidative medium/NaClO & 254.3 & 257.0 & $187.7^{*}$
\end{tabular}

*Evaluated from $150{ }^{\circ} \mathrm{C}$

**Evaluated from $120{ }^{\circ} \mathrm{C}$

packaging purposes. To this end, the stability of $\mathrm{PEO} / \mathrm{Alg}-$ Ag mats against sterilization and anti-mold procedures as well as against environmental changes was investigated by means of FESEM and TGA techniques. The analysis was 
Fig. 10 FESEM images

collected in direct configuration (in-lens mode) of PEO/Alg-Ag subjected to physical sterilization procedures. From top to bottom: heating at $120^{\circ} \mathrm{C}$ for $1 \mathrm{~h}$ (a), irradiation with UV light for $10 \mathrm{~min}(\mathbf{b})$ and $1 \mathrm{~h} \mathrm{(c)}$
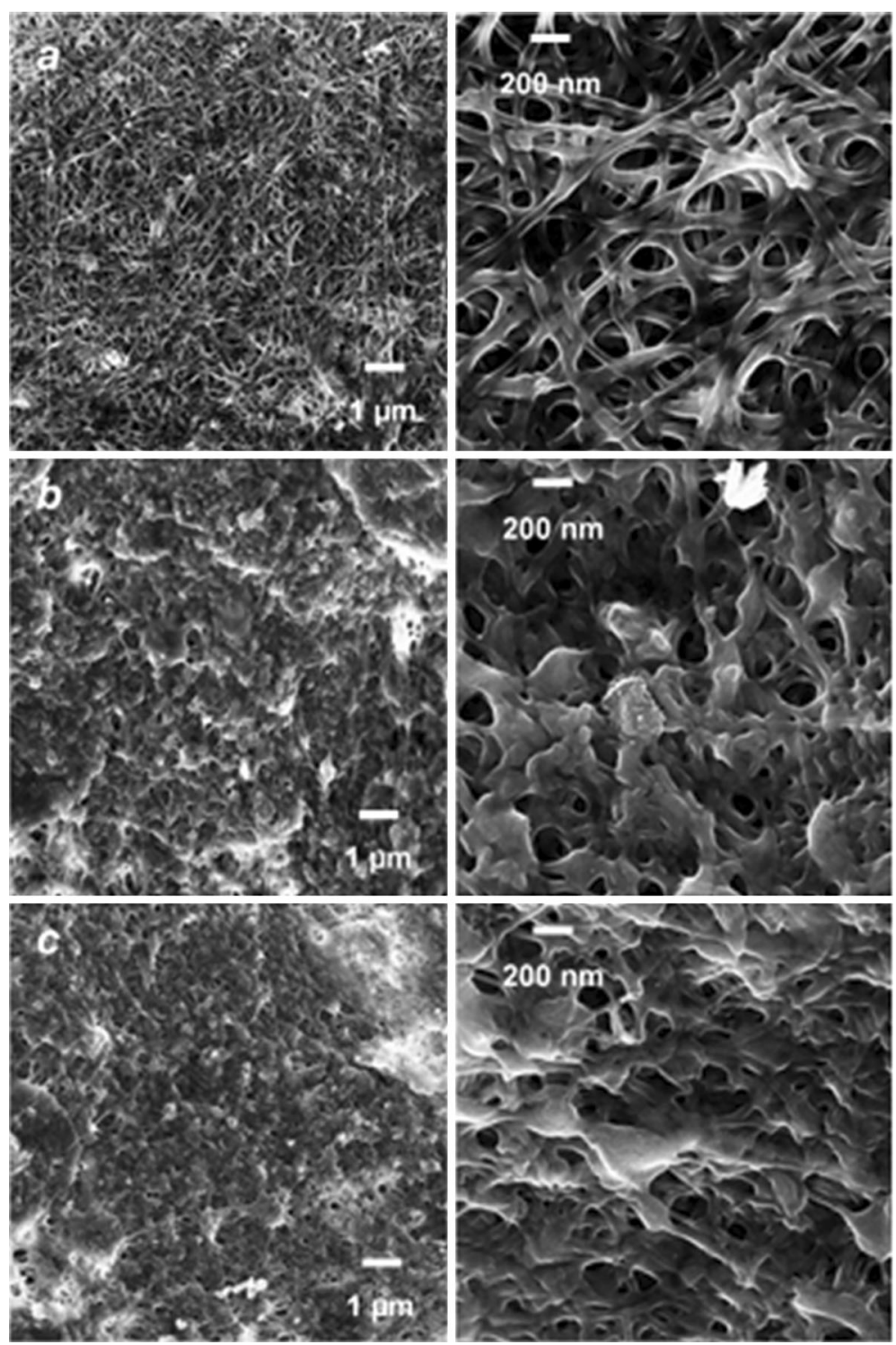

carried out under the drastic conditions detailed in "Experimental" section with the aim of testing the material behavior facing the most severe situations.

Figures 8 and 9 display the DTG curves obtained after the physical and chemical perturbations, respectively. In both cases, the curve of the untreated sample (green lines A) is added to facilitate the comparison. The thermal profiles show insignificant changes after treatments with temperature and UV light to indicate that sterilization by heat or high-energy irradiation is suitable for the composite nanofibers.

Different trends are instead observed following the chemical environmental changes. At strongly acidic $\mathrm{pH}$, the peak associated with PEO degradation almost disappears, whereas that attributable to the decomposition of alginate filled with AgNPs is just downshifted of about $15^{\circ} \mathrm{C}$ (Fig. 9, line B). This result suggests that only the polysaccharide component can resist to $\mathrm{HCl}$ solutions at high concentrations. At strongly alkaline $\mathrm{pH}$ or in the presence of aqueous $\mathrm{NaClO}$, acting as anti-mold sterilant solution (Fig. 9, lines $\mathrm{C}$ and D), the profiles corresponding to multi-steps mechanism are overall modified and entirely shifted under $300{ }^{\circ} \mathrm{C}$ to suggest that the thermal degradation of the nanofibers occurred at significantly lower temperature under these experimental conditions. It is likely that both alkaline and oxidant environments induced the breaking of the polymer chains, especially in the PEO component, and, consequently, the fragments degraded at lower temperatures.

The parameters usually employed to describe the thermal stability of materials are the thermal degradation temperature $\left(T_{\mathrm{d}}\right)$ and, for application purposes, the temperature at $5 \%$ mass loss $\left(T_{5 \%}\right)$. The values of $T_{\mathrm{d}}$ and $T_{5 \%}$ of the alginate moiety, extracted for untreated and treated 
PEO/Alg-Ag mats from DTG (Figs. 8, 9) and TG curves (Figs. 4S, 5S in electronic supplementary material), are collected in Table 3. $T_{5 \%}$ of the composite membranes was referred to dried samples and was obtained by properly rescaling the experimental profiles in order to exclude the mass loss associated to both physically and chemically absorbed water. The corresponding $T_{\mathrm{d}}$ of the alginate moiety evaluated under the same experimental conditions for all-polymeric PEO/Alg mats, taken as reference sample, is also reported to better highlight the AgNPs effect on the thermal properties of the polysaccharide-based nanocomposite.

Following the physical and chemical treatments, the $T_{\mathrm{d}}$ parameter displays the same decreasing trend for both samples, except for the 10-min and 1-h UV-irradiated PEO/Alg mats, which seemed to be stabilized over degradation after having been exposed to UV light, most likely because of cross-linking events occurred among the polysaccharide chains. In all other cases, the $T_{\mathrm{d}}$ values are comparable for the two membranes to underline once again that the presence of AgNPs does not significantly alter the thermal properties of alginate. As far as $T_{5} \%$ is concerned, also this parameter is not significantly affected by the physical perturbations, but exhibits relevant drops (from nearly $40{ }^{\circ} \mathrm{C}$ to over $100{ }^{\circ} \mathrm{C}$ ) when chemical environment changes occur, in agreement with the $T_{\mathrm{d}}$ behavior. However, it can be noted that the $T_{5 \%}$ values are always higher than $150{ }^{\circ} \mathrm{C}$ and then potentially compatible with biomedical and food applications.

As far as the post-treatment morphological characterization is concerned, Fig. 10 reports the FESEM images collected at two different magnifications for the composite mats subjected to the less impactful treatments, i.e., heating at $120{ }^{\circ} \mathrm{C}$ for $1 \mathrm{~h} \mathrm{(a)}$ and exposition to UV light for $10 \mathrm{~min}$ and $1 \mathrm{~h}$ (b and c, respectively). It is evident that whereas the heat did not alter the mats structure, UV irradiation induced changes in the membrane texture, which are better highlighted in the images at higher magnification. In detail, rearrangement of the nanofibers into spherical-like domains is observed with consequent reduction of the mat porosity, in terms of both pores size and number. The phenomena turned out to become even more noticeable as the exposure time to the electromagnetic radiation increases. Future investigation will be carried out to verify the effect of the morphology alterations on the mat properties.

\section{Conclusions}

This paper reports the preparation and characterization of a nanocomposite constituted by an alginate matrix embedding silver nanoparticles. The silver cores are synthesized directly in the polysaccharide bulk through a chemical reduction of the metal precursors carried out in aqueous solution. Once morphologically and spectroscopically characterized, the composite material, which turned out to be stable for months in aqueous suspension, was successfully processed with PEO by electrospinning technique to produce nanotextured mats mainly exploitable for biomedical applications and food industry. To this end, the stability of the composite nanofibers toward physical sterilization methods, anti-mold treatments and environmental perturbations was tested by studying the behavior of the mats under heating, UV irradiation, exposure to oxidative medium and acid-base changes by means of thermogravimetric analysis and morphological investigation. Under the severe experimental conditions adopted, the nanofibers were showed to be nearly unperturbed by physical treatments, opening the way to the use of heat or UV light as sterilization technique. Conversely, the membrane stability turned out to be more affected by chemical environments, especially when oxidizing or basic reagents are present. In any case, however, the thermal degradation of the polymeric matrix was found to occur well above the common operating temperatures for biomedical applications and food industry, which makes this nanocomposite material quite promising for tissue engineering or food packaging purposes.

On the whole, this work showed encouraging results about the possibility of preparing polysaccharide-based nanocomposite with intriguing physicochemical, antibacterial and processing properties through a simple and costeffective protocol. By taking into account the importance of employing renewable sources in biomedical and industrial fields, this bottom-up approach may represent a versatile route to develop biomaterials alternative to synthetic polymers.

Further studies will be devoted to test the effective antibacterial activity of the composite electrospun nanofibers as a function of the silver nanoparticles content.

\section{Electronic supplementary material}

Electronic Supplementary Material associated with this article is available free of charge at http://....Fig. 1S): Evolution with time of UV-Vis spectra of AgNPs in alginate solution; Fig. 2S): Size distribution histogram of AgNPs embedded in $\mathrm{PEO} / \mathrm{Alg}-\mathrm{Ag}$ electrospun mats deriving from FESEM investigation; Fig. 3S): EDS analysis of PEO/Alg/-Ag electrospun mats by FESEM investigation; Fig. 4S): TG curves of electrospun mats of PEO/ Alg-Ag before and after physical treatments; Fig. $5 \mathrm{~S}$ ): TG curves of electrospun mats of $\mathrm{PEO} / \mathrm{Alg}-\mathrm{Ag}$ before and after chemical treatments. 


\section{Compliance with ethical standards}

Conflict of interest All the authors declare that they have no conflict of interest.

\section{References}

1. Zafar R, Mahmood K, Tabasum S, Jabeen F, Noreen A, Zuber M. Polysaccharide based bionanocomposites, properties and applications: a review. Int J Biol Macromol. 2016;92:1012-24.

2. Zheng Y, Monty J, Linhart RJ. Polysaccharide-based nanocomposites and their applications. Carbohyd Res. 2015;405:23-32.

3. Cazón P, Velazquez G, Ramírez JA, Vázquez M. Polysaccharidebased films and coatings for food packaging: a review. Food Hydrocolloids. 2017;68:136-48.

4. Lee KY, Mooney DJ. Alginate: properties and biomedical applications. Prog Polym Sci. 2012;36:106-26.

5. Stewart MB, Gray SR, Vasiljevic T, Orbell JD. Exploring the molecular basis for the metal-mediated assembly of alginate gels. Carbohyd Polym. 2014;102:246-53.

6. Donati I, Holtan S, Morch YA, Borgogna M, Dentini M, SkjakBraek G. New hypothesis on the role of alternating sequences in calcium-alginate gels. Biomacromol. 2005;6:1031-40.

7. Stewart MB, Gray SR, Vasiljevic T, Orbell JD. The role of poly$\mathrm{M}$ and poly-GM sequences in the metal-mediated assembly of alginate gels. Carbohyd Polym. 2014;112:486-93.

8. Sikorski P, Mo F, Skjak-Braek G, Stokke BT. Evidence for eggbox-compatible interactions in calcium-alginate gels from fiber X-ray diffraction. Biomacromol. 2007;8:2098-103.

9. Vicini S, Castellano M, Mauri M, Marsano E. Gelling process for sodium alginate: new technical approach by using calcium rich micro-spheres. Carbohyd Polym. 2015;134:767-74.

10. Vicini S, Mauri M, Wichert J, Castellano M. Alginate gelling process: use of bivalent ions rich. Polym Eng Sci. 2017;57:531-6.

11. Velema J, Kaplan D. Biopolymer-based biomaterials as scaffolds for tissue engineering. Adv Biochem Eng Biotechnol. 2006;102:187-238

12. Rajkumar M, Meenakshisundaram N, Rajendran V. Development of nanocomposites based on hydroxyapatite/sodium alginate: synthesis and characterisation. Mater Charact. 2011;62:469-79.

13. Kumar A, Rao KM, Han SS. Development of sodium alginatexanthan gum based nanocomposite scaffolds reinforced with cellulose nanocrystals and halloysite nanotubes. Polym Test. 2017;63:214-25.

14. Liu S, Bastola AK, Li L. A 3D printable and mechanically robust hydrogel based on alginate and graphene oxide. ACS Appl Mater Interface. 2017;9:41473-81.

15. Malagurskia I, Levic S, Pantic M, Matijasevic D, Mitric M, Pavlovic V, Dimitrijevic-Brankovic S. Synthesis and antimicrobial properties of $\mathrm{Zn}$-mineralized alginate nanocomposites. Carbohyd Polym. 2017;165:313-21.

16. Travan A, Pelillo C, Donati I, Marsich E, Benincasa M, Scarpa T, Semeraro S, Turco G, Gennaro R, Paoletti S. Non-cytotoxic silver nanoparticle-polysaccharide nanocomposites with antimicrobial activity. Biomacromol. 2009;10:1429-35.

17. Gabriel JS, Gonzaga VAM, Poli AL, Schmitt CC. Photochemical synthesis of silver nanoparticles on chitosans/montmorillonite nanocomposite films and antibacterial activity. Carbohyd Polym. 2017;171:201-10.

18. Doshi J, Reneker DH. Electrospinning process and applications of electrospun fibers. J Electrostat. 1995;35:151-60.
19. Hayati I, Bailey A, Tadros TF. Investigations into the mechanism of electrohydrodynamic spraying of liquids: II. Mechanism of stable jet formation and electrical forces acting on a liquid cone. J Colloid Interface Sci. 1987;117:222-30.

20. Hohman MM, Shin M, Rutledge G, Brenner MP. Electrospinning and electrically forced jets. II. Applications. Phys Fluids. 2001;13:2221-36.

21. Reneker DH, Yarin AL, Fong H, Koombhongse S. Bending instability of electrically charged liquid jets of polymer solutions in electrospinning. J Appl Phys. 2000;87:4531-47.

22. Shin YM, Hohman MM, Brenner MP, Rutledge GC. Experimental characterization of electrospinning: the electrically forced jet and instabilities. Polymer. 2001;42:09955-67.

23. Taylor G. Electrically driven jets. Proc $R$ Soc A. 1969;313:453-75.

24. Castellano M, Cantù R, Mauri M, Marsano E, Vicini S. Poly(dimethylsiloxane)/TiO2 photocatalytic membranes obtained by different electrospinning systems. J Nanosci Nanotechnol. 2016;16:6587-94.

25. Huang L, Nagapudi K, Apkarian RP, Chaikof EL. Engineered collagen-PEO nanofibers and fabrics. J Biomater Sci Polym E. 2001;12:979-93.

26. Duan B, Dong CH, Yuan XY, Yao KD. Electrospinning of chitosan solutions in acetic acid with poly(ethylene oxide). J Biomater Sci Polym E. 2004;15:797-811.

27. Bonino CA, Krebs MD, Saquing CD, Jeong SI, Shearer KL, Alsberg E, Khan SA. Electrospinning alginate-based nanofibers: from blends to crosslinked low molecular weight alginate-only systems. Carbohyd Polym. 2011;85:111-9.

28. Lu JW, Zhu YL, Guo ZX, Hu P, Yu J. Electrospinning of sodium alginate with poly(ethylene oxide). Polymer. 2006;47:8026-31.

29. Seo SY, Lee GH, Lee SG, Jung SY, Lim JO, Choi JH. Alginatebased composite sponge containing silver nanoparticles synthesized in situ. Carbohydr Polym. 2012;90:109-15.

30. Zhao X, Li Q, Ma X, Quan F, Wanga J, Xia Y. The preparation of alginate-AgNPs composite fiber with green approach and its antibacterial activity. J Ind Eng Chem. 2012;24:188-95.

31. Alloisio M, Zappia S, Demartini A, Martinez Espinoza MI, Ottonelli M, Dellepiane G, Thea S, Cavalleri O, Rolandi R. Silver-polydiacetylene core-shell nanohybrids: from nano to mesoscale architectures. Nano-Struct Nano-Objects. 2015;4:15-22.

32. Drezner Z, Turel O. Normalizing variables with too-frequent values using a Kolmogorov-Smirnov test: a practical approach. Comput Ind Eng. 2011;61:1240-4.

33. Pham-Gia T, Hung TL. The mean and median absolute deviations. Math Comput Model. 2001;34:921-36.

34. Li J, He J, Huang Y, Li D, Chen X. Improving surface and mechanical properties of alginate films by using ethanol as a cosolvent during external gelation. Carbohyd Polym. 2015;123:208-16.

35. Salama A, Diab MA, Abou-Zeid RE, Aliohani HA, Shoueir KR. Crosslinked alginate/silica/zinc oxide nanocomposite: a sustainable material with antibacterial properties. Compos Commun. 2018;7:7-11.

36. Wu T, Huang J, Jiang Y, Hu Y, Te X, Liu D, Chen J. Formation of hydrogels based on chitosan/alginate for the delivery of lysozyme and their antibacterial activity. Food Chem. 2018;240:361-9.

37. Wang C, Luo W, Li P, Li S, Yang Z, Hu Z, Liu Y, Ao N. Preparation and evaluation of chitosan/alginate porous microspheres/Bletilla striata polysaccharide composite hemostatic sponges. Carbohyd Polym. 2017;174:432-42.

38. Choudharya S. Structural, optical, dielectric and electrical properties of (PEO-PVP)-ZnO nanocomposites. J Phys Chem Solids. 2018;121:196-209. 
39. Kiran KK, Ravi M, Pavani Y, Bhavani S, Sharma AK, Narasimha Rao VVR. Electrical conduction mechanism in $\mathrm{NaCl}$ complexed PEO/PVP polymer blend electrolytes. J Non-Cryst Solids. 2012;358:3205-11.

40. Huq T, Salmieri S, Khan A, Khan RA, Le Tien C, Riedl B, Fraschini C, Uribe-Calderon Bouchard J, Kamal MR, Lacroix M. Nanocrystalline cellulose (NCC) reinforced alginate based biodegradable nanocomposite film. Carbohyd Polym. 2012;90:1757-63.

41. Zille A, Fernandes MM, Francesko A, Tzanov T, Fernandes M, Oliveira FR, Almeida L, Amorim T, Carneiro N, Esteves MF, Souto AP. Size and aging effects on antimicrobial efficiency of silver nanoparticles coated on polyamide fabrics activated by atmospheric DBD plasma. ACS Appl Mater Interfaces. 2015;7:13731-44.

42. do Nascimento Porto Neto A, dos Santos Cruz CF, Russo Serafini M, dos Passos Menezes P, Barbosa Gomes de Carvalho YM, Santos Matos CR, Santos Nunes P, Cordeiro Cardoso J,
Cavalcanti de Albuquerque Junior RL, Rolim Neto PJ, Amaral Silva F, de Souza Araújo A. Usnic acid-incorporated alginate and gelatin sponges prepared by freeze-drying for biomedical applications. Carbohyd Polym. 2017;127:1703-13.

43. Guerra Nista SV, Bettini J, Innocentini Meia LH. Coaxial nanofibers of chitosan-alginate-PEO polycomplex obtained by electrospinning. Carbohyd Polym. 2015;127:222-8.

44. Cássia da Silva R, Gomes Cavalheiro ET. Synthesis, characterization, and thermal analysis of alginate and monoethanolamine product. J Therm Anal Calorim. 2015;120:855-62.

45. Laurenzio P, Malinconico M, Motta A, Vicinanza A. Synthesis and characterization of a novel alginate-poly(ethylene glycol) graft copolymer. Carbohyd Polym. 2005;62:274-82.

Publisher's Note Springer Nature remains neutral with regard to jurisdictional claims in published maps and institutional affiliations. 